\title{
Determinantes de género en el abordaje del dolor crónico
} Gender determinants in the approach to chronic pain

E. Gallach Solano, M. A. Bermejo Gómez, R. Robledo Algarra, R. M. Izquierdo Aguirre y M. A. Canos Verdecho Unidad de Dolor, Hospital Universitario y Politécnico La Fe. Valencia, España

\section{ABSTRACT}

The $20^{\text {th }}$ century has been an exceptional witness to the great advances and findings in the neurophysiological understanding and the multidisciplinary treatment of chronic pain. However, interest in the different form of expression in the painful perception between men and women and the specific psychological and social determinants associated with gender roles are relatively recent. In this article, an unsystematic review of the psychological and social differential determinants is carried out following the Neuromatrix model. Finally, the knowledge of those biases that we professionals have involuntarily is crucial to address the avoidable inequalities in treatment and the recovery of the health of men and women. Finally, recommendations are offered from the field of Clinical Psychology.

Key words: Choric pain, gender role, gender bias, psychological determinants of health, social determinants.

\section{RESUMEN}

El siglo $x x$ ha sido testigo de excepción de los grandes avances y hallazgos en la comprensión neurofisiológica y el tratamiento multidisciplicar del dolor crónico. No obstante, el interés por la forma diferente de expresión en la percepción dolorosa entre hombres y mujeres y los determinantes psicológicos y sociales específicos asociados a los roles de género es relativamente reciente. En este artículo se realiza una revisión no sistemática de los determinantes diferenciales psicológicos y sociales siguiendo el modelo de la neuromatriz. Por último, el conocimiento de aquellos sesgos que de forma involuntaria tenemos los profesionales es crucial para abordar las desigualdades evitables en tratamiento y la recuperación de la salud de hombres y mujeres. Finalmente se ofrecen recomendaciones desde el ámbito de la Psicología Clínica.

Palabras clave: Dolor crónico, rol de género, sesgos de género, determinantes psicológicos de salud, determinantes sociales.

\section{INTRODUCCIÓN}

El siglo $x x$ ha sido un testigo de excepción de los grandes avances en dolor crónico (DC): desarrollo de modelos neurofisiológicos y etiopatogénicos, multidisciplinariedad de la intervención, novedosas técnicas diagnósticas en neuroimagen, descripción precisa de síndromes específicos, métodos intervencionistas cada vez más eficaces, modelos complejos acerca de las implicaciones de factores psicológicos y sociales, parámetros de mejora en calidad, introducción de las nuevas $m$-health y de e-health... Todo ello ha generado un modelo asistencial cada vez más sofisticado, personalizado y eficaz. No obstante, tal como señala Ruiz-Cantero (1), en la era de la genómica y de una atención cada vez más personalizada, durante mucho tiempo se ha ignorado la influencia del género. Se parte de la presunción errónea de igualdad entre mujeres y hombres en el curso de la enfermedad, es decir, en la expresión de las enfermedades (signos y síntomas), en la respuesta a los tratamientos y en los pronósticos. Recientemente Miller, Kararigas y Seeland [?] 
destacaron que, siendo el sexo una variable fundamental de la fisiología humana, rara vez se considera en el diseño de estudios fisiológicos básicos. De hecho, científicos de nuestro ámbito confiesan que la investigación con cobayas hembras, hormonalmente inestables, introduce variables de confusión en los diseños. La consecuencia de esta paradójica situación fueron errores y demoras en el proceso diagnóstico y en la estrategia terapéutica y pronósticos inciertos. Identificar las diferencias sexuales y los comportamientos diferentes de mujeres y hombres, determinados por estereotipos (normas y valores) incorporados durante la socialización, que tienen impacto en el cuerpo (células, hormonas y órganos), y viceversa, permite identificar las diferencias en la expresión de los estados de salud-enfermedad.

La biología no miente: hombres y mujeres somos diferentes, el funcionamiento es distinto, tenemos estructuras, órganos, fisiología diferente y un despliegue hormonal claramente responsable de un modo de interactuar, pensar, sentir y emocionarnos que nos asemeja más a nuestros congéneres. También los cambios sociales y culturales nos hacen recorrer itinerarios vitales complementarios, pero diferentes. Sin embargo, a pesar de este reconocimiento irrefutable, la incorporación de la visión diferencial en las Ciencias de la Salud es relativamente reciente. Sensibles a esta realidad, hace solo 25 años investigadores, epidemiólogos y clínicos del ámbito del DC evidenciaron que hombres y mujeres responden de forma diferente al dolor, situando una mayor prevalencia en las mujeres. Un hecho relevante fue la publicación en 2007 realizada por el Grupo de Trabajo de Sexo, Género y Dolor de la Asociación Internacional para el Estudio del Dolor (IASP) de un exhaustivo trabajo sobre variables físicas y psicosociales que impactan en el dolor y establecieron una serie de recomendaciones centradas en el uso del modelo biopsicosocial para el abordaje global del dolor, incluyendo factores físicos, cerebrales, psicológicos, hormonales y culturales y su repercusión diferencial en función del género [3].

Por otro lado, encuestas publicadas por la Sociedad Española de Dolor (SED), el Observatorio del Dolor y el Ministerio de Sanidad [4], junto a las realizadas por la Plataforma de Organizaciones de Pacientes, coinciden al señalar que la prevalencia de dolor crónico es al menos dos veces mayor en mujeres respecto a varones, siendo este más frecuente, intenso y de mayor duración. Indican que las mujeres están sobrerrepresentadas en ciertas patologías como la fibromialgia, las algias cráneo-faciales, pero que estas diferencias tienden a minimizarse a partir de los 85 años. También indican que son ellas las que declaran tener peor salud y solicitan ayuda con mayor frecuencia, consumiendo más analgésicos prescritos y no prescritos que los varones (5). Parece pues, que los mecanismos del dolor operan de forma diferente entre hombres y mujeres.

\section{SI MELZACK LEVANTARA LA CABEZA: LA PERSPECTIVA DE GÉNERO EN LA NEUROMATRIZ}

¿Por qué somos diferentes? Se ha tratado de dar respuesta desde diversas disciplinas. Recientes estu- dios neurofisiológicos han constatado que existe una respuesta a la analgesia y al estrés y mayor sensibilidad al dolor entre las mujeres. Todo esto se atribuye a diferencias anatomofisiológicas entre hombres y mujeres (hipocampo de mayor tamaño, nivel de activación de amígdala y corteza prefrontal, menor afinidad en receptores glucocorticoides] y también neuroendocrinas. El estrógeno, la progesterona y otras hormonas gonadales tienen una función compleja en los procesos inflamatorios y en la respuesta al dolor implicando cambios en umbral, percepción y tolerancia al dolor y respuesta analgésica [6] entre hombres y mujeres.

Desde la perspectiva psicosocial, se ha demostrado que los factores psicosociales contribuyen sustancialmente a la percepción del dolor y pueden influir diferencialmente entre hombres y mujeres [7]. Parece existir un acuerdo implícito de que las mujeres se encuentran más expuestas y presentan mayor vulnerabilidad al dolor por razón de género [8]. Esta vulnerabilidad se relaciona con algunos factores sociales que perpetúan un patrón de desigualdad y consolidan la brecha de género: exposición temprana al estrés ambiental, físico y mental, historia previa de dolor, antecedentes familiares de dolor (incrementan la sensibilidad al dolor en las mujeres, no en varones], traumatismos físicos o mentales, largos periodos de sueño interrumpido y de mala calidad (periodo de lactancia y crianza), trabajos repetitivos, monótonos, sin condiciones ergonómicas aceptables a edades muy tempranas [9], dificultad para la autorrealización personal y profesional más allá de la crianza, desempeño de rol de cuidadora simultáneo al rol profesional y doméstico, falta de soporte familiar, problemas de conciliación, etc. Además, existen otros factores subjetivos relacionados con la experiencia vivida, como experiencias infantiles traumáticas, violencia, desarraigo, pérdidas, desvalorización de roles femeninos, ideales de feminidad y masculinidad inalcanzables, de mayor incidencia en las mujeres que también contribuyen a la desigualdad. Dos de los factores más investigados relacionados con dolor es el abuso físico y sexual, problema estructural de gravedad que se asocia con mayores tasas de dolor pélvico en las mujeres (4).

Considerando el modelo de la neuromatriz de Melzack [10] y las diferentes dimensiones del dolor, las investigaciones indican que en las mujeres existe un mayor predominio del componente cognitivo-evaluativo (por qué me duele, cómo influye en mi forma de ver el mundo, en mis creencias y valores] y afectivo-motivacional del dolor (cómo afecta el dolor a mi vida, si amenaza mi supervivencia, mi calidad de vida, disminuye mi autoeficacia). Frente a ello, en los varones se produce una preponderancia de la dimensión sensorial-discriminativa [cuánto me duele y qué cosas puedo hacer o debo dejar de hacer con esta molestia] $[6,11]$.

Se ha constatado también que existe una mayor presencia de trastornos comórbidos. La ansiedad y la depresión relacionados con procesos dolorosos es frecuente, próximo al $20 \%$ según refiere el Observatorio del Dolor y la SED (4), y se genera tanto en varones como en mujeres cuando la intensidad del dolor es alta y especialmente en ellos, cuando se produce una disminución de la actividad laboral o genera importante discapacidad [6]. 
Sin embargo, son ellas las que solicitan ayuda médica con más frecuencia. Aquí influyen también estereotipos sociales de género (heroico y estoico del varón frente al vulnerable y aceptable para las mujeres] que generan creencias en pacientes e incluso en los profesionales sobre "los hombres no lloran", no es socialmente aceptable hablar de dolor; "la mujer es vulnerable", es un tema frecuente de conversación y está reconocido socialmente $][5,12,13]$.

Los trastornos mentales comunes (depresión, ansiedad y somatizaciones] son el doble de frecuentes en mujeres que en hombres. Ello no parece debido a factores biológicos ligados al sexo, que aunque puedan existir no justifican el resultado. Por el contrario, influyen factores psicosociales relacionados, en parte, con el papel de género tradicional que expone a las mujeres a mayores tensiones (el cuidado, aislamiento, dependencia, situaciones de subordinación, falta de proyecto y tiempo propios...) al tiempo que les resta capacidad de acción y autonomía para modificar su entorno estresante, además de la exposición a violencia de género; y en parte debidos a los modelos de transición de género, por acumulación de roles antiguos y nuevos, con la consecuente sobrecarga psicosocial. Rodríguez (14) evidenció que existe un desarrollo progresivo y lineal de la presencia de dolor lumbar en varones conforme aumenta la edad, mientras que en las mujeres se observa un crecimiento exponencial entre los 30 y 50 años de edad, tras lo cual se evidencia una meseta y posterior caída. En una investigación desarrollada por Harutyunyan y cols. (13), y presentada a la Sociedad Española de Dolor, en una muestra de pacientes con dolor crónico de una Unidad de Dolor, no se encontraron diferencias en cuanto al sexo en intensidad del dolor en términos generales, pero si el dolor era de origen neuropático, ellas presentan dolor más intenso. En este mismo año, con una muestra de dolor lumbar, Ramos (15) señaló que este es más prevalente en varones y lo atribuye al desempeño laboral. Todo ello nos sugiere que los factores psicosociales y de género tienen una gran relevancia en el origen, mantenimiento y resolución del dolor crónico.

También se han declarado diferencias respecto a las estrategias de afrontamiento utilizadas para el automanejo del dolor. En este sentido, las mujeres utilizan estrategias más adaptativas, centradas en el apoyo social, reevaluaciones positivas y autorregulación emocional, y esto proporciona mayor sensación de control y cierta habituación a la intensidad del dolor (16). Frente a ellas, los varones prefieren farmacoterapia o técnicas de afrontamiento pasivo como la distracción o activas como centradas en la resolución del problema. Por ello son mejores respondedores y más adherentes al ejercicio físico, fisioterapia o rehabilitación, que las mujeres. En este mismo sentido, los varones prefieren modalidades terapéuticas centradas en el ejercicio físico o aquellas terapias que incluyan componentes conductuales de manejo del dolor. Sin embargo, ellas responden mejor frente a modalidades cognitivo-conductuales y de autorregulación emocional. Sin embargo, si se trata de terapia multicomponente (cognitiva, emocional y conductual] no se aprecian diferencias significativas entre ambos géneros (17-19).

\section{SESGOS DE GÉNERO EN LOS PROFESIONALES SANITARIOS}

Los roles de género, mediatizados por patrones culturales y creencias, influyen sobre la conducta y los individuos respondemos según el propio rol interiorizado. Estas diferencias, como no podría ser de otro modo, también se reflejan en la percepción de la enfermedad y del dolor. Los roles de género, de este modo, interfieren en la percepción del dolor y en su cronificación, tal y como hemos descrito. Los antecedentes androcéntricos históricos de las disciplinas en Ciencias de la Salud han producido resistencias a la evidente necesidad de incorporar el enfoque de género, generando barreras individuales e interpersonales que conducen, finalmente, a la existencia de sesgos en el propio proceso asistencial. Y por tanto, influyen tanto en los pacientes que sufren dolor como en los profesionales que lo tratamos de forma sesgada, quizás sin saberlo, en función de nuestro propio modelo de funcionamiento profesional.

En una investigación realizada en 2004 (20) se identificaron los siguientes sesgos de género atribuibles a los profesionales:

1. Sesgo de género en la utilización hospitalaria. La bibliografía científica informa que la proporción de varones ingresados en el hospital es superior al de las mujeres. Esta no es solo responsabilidad de los pacientes, sino que está mediada por el médico y el sistema sanitario. Con relación a los tratamientos sintomáticos, como el dolor, en Cataluña se observó en 1964 personas (un $50,3 \%$, mujeres] que en cualquier grupo de edad las mujeres acuden más al médico y se automedican más que los hombres, pero los hombres reciben más tratamiento hospitalario que las mujeres.

2. Sesgos de género en la aplicación de procesos terapéuticos. Pese a no detectar diferencias significativas en la presentación de infartos, las mujeres presentan al ingreso cuadros de mayor severidad (el $14 \%$ de los hombres y el $21 \%$ de las mujeres]. También se observa un mayor esfuerzo terapéutico en hombres, medido en términos de acceso a la unidad de cuidados intensivos (UCI) (el 90 frente al $75 \%$ ). Aunque estos resultados no miden costes absolutos, sirven para hacer una comparación por sexos. Estos resultados son congruentes con los observados en otras especialidades. Hasta el momento, no existen datos específicos disgregados por género sobre coste farmacéutico o intervencionista en las Unidades de Dolor, y si bien parece existir una demanda de 3 a 1 en el tratamiento, esta tendencia se mantiene respecto a la pauta intervencionista. No obstante, sería conveniente tener en cuenta estos factores para poder prevenir actuaciones sesgadas.

3. Sesgo de género en la prescripción y consumo de psicofármacos. Se constata un mayor consumo y prescripción de psicofármacos en mujeres. Ello puede reflejar una mayor prevalencia de depresión y ansiedad en estas, o que al quejarse induzcan la prescripción más que los hombres. Pero también es posible que los facultativos atribuyan los síntomas físicos (o de presentación 
atípica) a factores psicológicos más fácilmente en las mujeres que en los hombres, o tiendan a prescribir fármacos para síntomas depresivos de baja intensidad a las mujeres más que a los hombres. Lo destacable es que el malestar emocional de las mujeres está excesivamente medicalizado y sería recomendable desde las Unidades de Dolor atender a estos factores desde una perspectiva más psicosocial.

\section{RECOMENDACIONES Y CONCLUSIONES}

Los hallazgos obtenidos hasta el momento clarifican algunos aspectos: establecen que el dolor crónico es más prevalente en mujeres, que estas presentan mayor riesgo de desarrollarlo y que presentan un umbral doloroso más bajo, con mayor sensibilidad al dolor dependiendo del tipo de estímulo doloroso, especialmente de presión o térmico. Sin embargo, presentan una mayor habituación al mismo, especialmente si los estímulos dolorosos se mantienen en el tiempo. También presentan mayor número de localizaciones dolorosas, como en el caso de la fibromialgia con una ratio de 20 mujeres por cada varón. Sin embargo, si determinamos la intensidad, las diferencias entre géneros aparecen sobre dolor intenso, no sobre leve y moderado. Respecto a la naturaleza del dolor, existen algunas patologías dolorosas típicamente femeninas: fibromialgia, cefaleas migrañosas y tensionales.

En cualquier caso, la intervención desde las Unidades de Dolor es necesario considerar algunos principios básicos, útiles tanto para la intervención desde la perspectiva de género como desde una perspectiva más biopsicosocial (psicopatológica), la herramienta básica siempre será la relación facultativo/paciente:

- La consideración de la situación personal y social del paciente como única. Atención centrada en la persona y no en la enfermedad o el médico (incluye su biografía, el contexto personal y social].

- La identificación del modelo social y de los roles de género asociados (factores de riesgo y protectores].

- En la expresión de la percepción dolorosa puede existir predominio de una dimensión sobre las demás (la emocional o cognitiva sobre la perceptivo- evaluativa, por ejemplo]. También en la terapéutica es necesario barajar esta triple opción.

- La relación de confianza con el/la paciente favorece el empoderamiento en automanejo del dolor y la adhesión a estrategias de afrontamiento no farmacológica que requieran su participación: prescripción de ejercicio físico y descenso ponderal, adiestramiento en técnicas de autorregulación emocional, etc.

Por último, mencionamos algunas claves que pueden contribuir a reducir la brecha de género en el abordaje del dolor crónico:

- Cuidar la estratificación en las investigaciones de acuerdo con criterios epidemiológicos y clínicos, incluyendo la variable género como realidad a investigar en los pacientes con DC.

- Aceptar la diferencia en la percepción, sensibilidad, intensidad, duración, evolución, pronóstico y tratamiento del dolor crónico entre varones y mujeres.

- Incorporar la mirada biopsicosocial durante todo el proceso diagnóstico y terapéutico, abordando los factores psicosociales asociados al dolor tanto en varones como en mujeres.

$Y$ ya para finalizar y a modo de reflexión, el conocimiento de sesgos de género en medicina es crucial para abordar las desigualdades evitables en la recuperación de la salud de las mujeres. Se han producido avances significativos en el conocimiento de fisiología, etiopatogenia, farmacoterapia y tratamientos intervencionistas cada vez más eficaces e individualizados en el ámbito del dolor crónico. También se han producido importantes cambios estructurales en el contexto de roles de género y logros sociales, laborales, micro y macroeconómicos y culturales para atenuar la desigualdad, que sin duda repercutirán en modelos de salud-enfermedad también diferentes y complejos. Parece pues, que todavía nos queda un largo camino por recorrer en el ámbito de tratamiento diferencial y deberemos asumir esta gran responsabilidad social, sanitaria y científicamente compartida para poder dar una respuesta a la nueva realidad diferencial de dolor y género.

\section{CONFLICTO DE INTERESES}

Todas y cada una de las autoras declara carecer de conflicto de intereses en este manuscrito.

\section{BIBLIOGRAFÍA}

1. Ruiz-Cantero MT, Tomás-Aznar C, Rodríguez-Jaume MJ, Pérez-Sedeño E, Gasch-Gallén A. Agenda de género en la formación en ciencias de la salud: experiencias internacionales para reducir tiempos en España. Gac Sanit. 2020;33(5):48590. DOI: 10.1016/j.gaceta.2018.03.010.

2. Miller VM, Kararigas G, Seeland U, Regitz-Zagrosek V, Kublickiene $\mathrm{K}$, Einstein $\mathrm{G}$, et al. Integrating topics of sex and gender into medical curricula - lessons from the international community. Biol Sex Differ. 2016;7(Suppl 1):44. DOI: 10.1186/ s13293-016-0093-7.

3. Greenspan JD, Craft RM, LeResche L, Arendt-Nielsen L, Berkley KJ, Fillingim RB, ... et al. Studying sex and gender differences in pain and analgesia: a consensus report. Pain. 2007;132(Suppl. 1):S26-S45. DOI: 10.1016/j.pain.2007. 10.014.

4. Encuesta Nacional de Salud ENSE, España 2017 [Internet]. Ministerio de Sanidad, Consumo y Bienestar Social. 2017; [citado 12 de marzo de 2020]. Disponible en: https://www. mscbs.gob.es/estadEstudios/estadisticas/encuestaNacional/encuesta2017.htm

5. Mapplebeck JC, Beggs S, Salter MW. Sex differences in pain: a tale of two immune cells. Pain. 2016;157(Suppl. 1):S2-S6. DOl: 10.1097/j.pain.0000000000000389.

6. Fillingim RB. Sex, gender, and pain. In: Principles of GenderSpecific Medicine. Legato M (ed). Academic Press; 2017. p. 481-96.

7. Gatchel RJ, Mayer TG, Kidner CL, McGeary D. Are gender, marital status or parenthood risk factors for outcome of treatment for chronic disabling spinal disorders? J Occup 
Rehabil. 2005;15(2):191-201. DOI: 10.1007/s10926005-1218-8.

8. Defrin R, Shramm L, Eli I. Gender role expectations of pain is associated with pain tolerance limit but not with pain threshold. Pain. 2009;145(1-2):230-6. DOI: 10.1016/j. pain.2009.06.028.

9. Wijnhoven HA, de Vet HC, Picavet HSJ. Explaining sex differences in chronic musculoskeletal pain in a general population. Pain. 2006;124(1-2):158-66. D0l: 10.1016/j.pain.2006.04.012.

10. Melzack R, Casey KL. Sensory, motivational, and central control determinants of pain: a new conceptual model. In: The skin senses. Kensalo DR, ed. Springfield: Charles C. Thomas; 1968. p. 423-43.

11. Fillingim RB. Individual differences in pain: understanding the mosaic that makes pain personal. Pain. 2017;158(Suppl 1):S11-8. DOl: 10.1097/j.pain.0000000000000775.

12. Bartley EJ, King CD, Sibille KT, Cruz-Almeida Y, Riley III JL, Glover $T L$, et al. Enhanced pain sensitivity among individuals with symptomatic knee osteoarthritis: potential sex differences in central sensitization. Arthritis Care Res. 2016;68(4):47280. DOl: 10.1002/acr.22712.

13. Harutyunyan A, Pastor JS, Dolz VM, Martí AM. Diferencias de género en pacientes con dolor crónico. Comunicación presentada al XII Congreso de la SED.
14. Rodríguez A. Revisión de factores psicológicos y estrategias para el manejo del dolor crónico lumbar desde diferentes modelos teóricos. Dolor. 2014;23(62):24-34.

15. Ramos Rangel Y, Santana Morfa AR, Valladares González AM, López Angulo L, González Brito M. Relación entre estados emocionales y variables clínicas en pacientes con dolor crónico lumbar. Rev Cubana Med Gen Integr. 2017;33(2):180-90.

16. Cook AJ, Chastain DC. The classification of patients with chronic pain: age and sex differences. Pain Res Manag. 2001;6(3):142-51. DOl: 10.1155/2001/376352.

17. Bartley EJ, Fillingim RB. Sex differences in pain: A brief review of clinical and experimental findings. Survey Anesthesiology. 2016;60(4):175-6. DOI: 10.1097/01. sa.0000484819.20819.8b.

18. Bartley EJ, Palit S. Gender and pain. Curr Anesthesiol Rep. 2016;6(4):344-53. DOI: 10.1007/s40140-0160177-2.

19. Turk DC, Monarch ES. Biopsychosocial perspective on chronic pain. In: D. C. Turk \& R. J. Gatchel (Eds.). Psychological approaches to pain management: A practitioner's handbook. The Guilford Press; 2018. p. 3-24.

20. Ruiz-Cantero MT, Verdú-Delgado M. Sesgo de género en el esfuerzo terapéutico. Gac Sanit. 2004;18(4):118-25. 